\section{Intraosseous fluid resuscitation in meningococcal disease and lower limb injury}

\author{
Suparna Dasgupta,' Stephen D. Playfor ${ }^{2}$ \\ 'Royal Manchester Children's Hospital, \\ Oxford Road, Manchester, UK; \\ 2Paediatric Intensive Care Unit, \\ Royal Manchester Children's Hospital, \\ Oxford Road, Manchester, UK
}

\begin{abstract}
We set out to review the recent incidence of extravasation and compartment syndrome in children with meningococcal disease admitted to our Paediatric Intensive Care Unit (PICU) who had been resuscitated with intraosseous (I0) needles. Over a 12-month period, 18 children were admitted to PICU with meningococcal sepsis. Four of these children were resuscitated with IO needles and 2 developed serious complications as a result of extravasation and compartment syndrome. Clinical practice guidelines for children with severe sepsis advocate aggressive early fluid resuscitation therapy. We have identified extravasation and lower limb injury as a potential issue of increasing concern with appropriate aggressive IO fluid resuscitation in severe septic shock in children. Powered IO access systems such as the EZ-IO system offer advantages in terms of placement speed, accuracy and bone entry site profile at minimal extra financial cost
\end{abstract}

\section{Introduction}

Meningococcal disease (MCD) continues to be the most common infective cause of death in children in the United Kingdom. In cases of severe septic shock, children frequently require large volumes of fluid resuscitation and when faced with difficult vascular access, the intraosseous (IO) route offers a prompt and effective route through which to infuse resuscitation fluids and drugs. Extravasation is a well recognised complication of IO access leading to limb-threatening events.

Over a 12-month period, 18 children with meningococcal sepsis were admitted to our Paediatric Intensive Care Unit (PICU). Four of these children had I0 needles inserted in their referring hospitals and 2 developed serious complications as a result of these. The local ethics committee has approved this paper for publication.

\section{Case \#1}

A 20-month old girl presented to her local Emergency Department with severe MCD. She had an I0 needle sited in her right tibia during initial resuscitation and she received in total $120 \mathrm{ml} / \mathrm{kg}$ of crystalloid boluses and $30 \mathrm{~mL} / \mathrm{kg}$ of colloid boluses prior to transfer to the PICU. Her right lower leg became increasingly swollen and tense; 15 hours after admission a diagnosis of compartment syndrome was made and fasciotomy of all lower limb compartments was carried out.

Six days after admission she underwent debridement of her right lower leg which was repeated on day 8 . When she was taken to theatre for skin grafting on day 11, the tibia was noted to be necrotic (Figure 1) and a bone scan confirmed avascular necrosis and shortening of the tibia. Two months later radiological assessment of the affected leg revealed widespread skeletal changes with periosteal new bone formation along the tibial shaft but with no residual osteomyelitis.

\section{Case \#2}

A 5-month old girl presented to her local Emergency Department with severe MCD. She required bilateral tibial $\mathrm{IO}$ line insertion during her initial resuscitation and received 120 $\mathrm{mL} / \mathrm{kg}$ of resuscitation fluid $(0.9 \%$ saline and colloids) through these lines along with bolus doses of $50 \%$ glucose solution, calcium gluconate $10 \%$ and sodium bicarbonate $8.4 \%$. Extravasation was noted in the Emergency Department and within two hours of presentation both lower limbs were swollen and tense. Immediate bilateral fasciotomy of all lower limb compartments was carried out but unfortunately perfusion of her legs continued to deteriorate and she eventually required bilateral below knee amputation two weeks later.

\section{Discussion}

The technique of IO needle insertion was initially described in the 1920s and 1930s when investigators conducting bone marrow transplant research noted that the marrow cavity of a long bone was a promising site for vascular access. During World War II, IO access, particularly of the sternum, was reportedly widespread but after the 1940s, during an era in which there was little pre-hospital care, the technique fell out of routine use. Interest was rekindled in the 1980s and since then the technique has become commonplace during the care of critically ill children, particularly during
Correspondence: Stephen D Playfor, Paediatric Intensive Care Unit, Royal Manchester Children's Hospital, Oxford Road, Manchester M13 9WL, UK. E-mail: stephen.playfor@cmft.nhs.uk

Key words: meningococcal disease, intraosseous, complications.

Contributions: both authors contributed to study conception and design, and the acquisition and interpretation of data. Both authors drafted the article, revised it together for important intellectual content, and approved the final version to be published.

Conflict of interest: the authors report no conflicts of interest.

Received for publication: 24 June 2009.

Revision received: 24 September 2009.

Accepted for publication: 13 October 2009.

This work is licensed under a Creative Commons Attribution 3.0 License (by-nc 3.0).

(C) Copyright S. Dasgupta and S.D. Playfor, 2010

Licensee PAGEPress, Italy

Pediatric Reports 2010; 2:e5

doi:10.4081/pr.2010.e5

their initial resuscitation.

Potential complications of IO access include failure of the technique due to the device or the operator, extravasation injury, compartment syndrome with or without limb amputation, ${ }^{2}$ bone infections, although a meta-analysis review of 4,270 cases of IO use found only 27 cases of osteomyelitis $(0.63 \%)$, fat embolism, skin necrosis, growth plate damage and fractures. Risk of injury to the growth plates has been shown to be small by following serial tibial measurements in children aged one year and over after having an IO needle placed with no clinically significant difference between control and infusion leg lengths.

Over the last decade there has been a significant improvement in mortality rates of children with MCD. The reasons for this are multifactorial, but one of the major contributory factors has been recognition of the benefit of aggressive early fluid resuscitation and inotrope therapy. Clinical practice guidelines for children with severe sepsis produced by Carcillo et al. ${ }^{3}$ advocate the administration of 'up to and over $60 \mathrm{~mL} / \mathrm{kg}$ ' of resuscitation fluid within 15 minutes of arrival in the Emergency Department. A specific early management algorithm for MCD which has been extensively distributed and utilized throughout Emergency Departments and Paediatric units in the UK has been recently updated. ${ }^{4}$ This algorithm also recommends aggressive fluid resuscitation therapy with initial $20 \mathrm{~mL} / \mathrm{kg}$ boluses of colloid or crystalloid solutions each given over 5-10 minutes. 


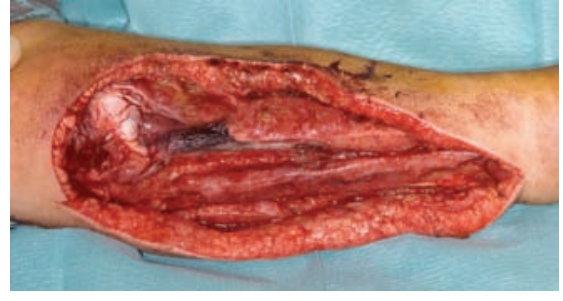

Figure 1. Tibial osteomyelitis.

(A) Manual I0 insertion

(B) EZ-IO insertion
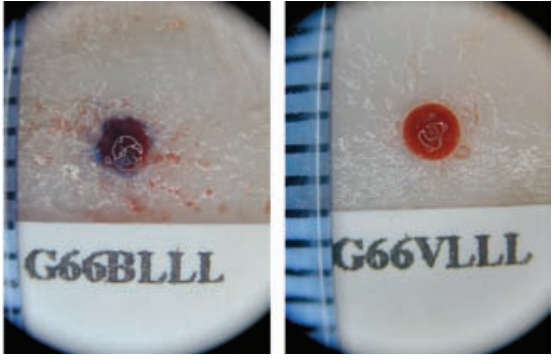

Figure $2 \mathrm{~A}$ and B. Entry sites in bone samples following IO access.

There is concern that the rate of complications of IO access, particularly extravasation injury and compartment syndrome, may increase in children with severe septic shock as patients appropriately receive larger volumes of resuscitation fluid through these lines. In a recent study of pediatric IO lines, Fiorito documented complications in 7 of $58 \mathrm{IO}$ placements (12\%), all limited to local edema or infiltration; it should be noted, however, that only $11 \%$ of patients in this study had a diagnosis of sepsis and as such the majority of the children included in this study will not have received large volumes of fluid intraosseously.

Manual insertion of $\mathrm{IO}$ needles results in an irregular entry site into bone (Figure 2A) mak- ing extravasation more likely. In contrast, powered or assisted IO access systems can provide more rapid access to the vascular compartment and result in a very regular entry site, minimizing the risks of extravasation (Figure 2B).

The EZ-IO system by Vidacare consists of a small hand-held battery-powered drill device and bevelled, hollow drill-tipped needles which may be quickly and easily placed in children and adults. We have used this system with success in the PICU and during the retrieval of critically ill children. Depth of needle insertion is accurately controlled and IO infusion can usually be commenced within 60 seconds of starting the procedure. The reusable EZ-IO drill costs around £190, whilst the EZ-IO needles are of a similar price to standard handheld IO needles. In a recent prospective multicenter study of 250 adult patients in the prehospital setting, the EZ-IO system was deployed with a $97 \%$ success rate, with a mean insertion time of ten seconds and with a mean pain score in alert patients of 3.8 (on a 1-10 visual analog scale). ${ }^{5}$

Other assisted IO access systems include the Bone Injection Gun (BIG) and the FAST1 system. The BIG by WaisMed comes in a range of sizes including a pediatric model; it is spring loaded and automatically fires a needle directly into the bone. Anecdotal reports have linked the deployment of this device with tibial fractures. ${ }^{6}$ The FAST1 system by the Pyng Medical Corporation is a specific sternal IO device for adults, which has been most widely used in defens medicine.

\section{Conclusions}

There is little published data available on changes in the pattern of morbidity due to MCD. Given the improvements in overall mortality we have seen over recent years, there will inevitably be a greater focus on the quality of life of survivors, with survival being at minimum cost rather than at any cost. We have identified extravasation and lower limb injury as a potential issue of increasing concern with appropriate aggressive I0 fluid resuscitation in severe septic shock in children. Powered IO access systems such as the EZ-IO system offer advantages in terms of placement speed, accuracy and bone entry site profile at minimal extra financial cost. Systems such as the EZ-IO warrant formal evaluation to assess their safety and complication profiles.

\section{References}

1. Simmons CE, Johnson NE, Perkins RM, et al. Intraosseous extravasation complication reports. Ann Emerg Med 1994;23:36366.

2. Launay F, Paut 0 , Katchburian M, et al. Leg amputation after intraosseous infusion in a 7-month-old infant: a case report. J Trauma 2003;55:788-90.

3. Carcillo JA, Fields AI: American College of Critical Care Medicine Task Force Committee Members. Clinical practice parameters for hemodynamic support of pediatric and neonatal patients in septic shock. Crit Care Med 2002;30:1365-78.

4. Pollard AJ, Nadel S, Ninis $\mathrm{N}$, et al. Emergency management of meningococcal disease: eight years on. Arch Dis Child 2007;92:283-86.

5. Davidoff J, Fowler R, Gordon D, et al Clinical evaluation of a novel intraosseous device for adults: prospective, 250-patient, multi-center trial. JEMS 2005;30:s20-3.

6. Bowley DM, Loveland J, Pitcher GJ. Tibial fracture as a complication of intraosseous infusion during pediatric resuscitation. $\mathrm{J}$ Trauma 2003;55:786-87. 